\title{
Rapid Visible Improvements in Onychomycosis: An Open-Label Clinical Investigation of Kerasal Nail $^{\text {TM }}$
}

\author{
Jiun Yit Pan1, Chris Lixian Tan², Anders Toft ${ }^{3 *}$ \\ ${ }^{1}$ National Skin Centre, Singapore City, Singapore \\ ${ }^{2}$ Division of Dermatology, National University Hospital, Singapore City, Singapore \\ ${ }^{3}$ Moberg Pharma AB, Bromma, Sweden \\ Email: *anders.toft@mobergpharma.se
}

How to cite this paper: Pan, J.Y., Tan, C.L. and Toft, A. (2017) Rapid Visible Improvements in Onychomycosis: An Open-Label Clinical Investigation of Kerasal Nail ${ }^{\mathrm{TM}}$. Journal of Cosmetics, Dermatological Sciences and Applications, 7, 57-66. https://doi.org/10.4236/jcdsa.2017.71006

Received: February 4, 2017

Accepted: March 10, 2017

Published: March 13, 2017

Copyright $\odot 2017$ by authors and Scientific Research Publishing Inc. This work is licensed under the Creative Commons Attribution International License (CC BY 4.0).

http://creativecommons.org/licenses/by/4.0/ (c) (i) Open Access

\begin{abstract}
Traditional treatments for onychomycosis, a fungal disease primarily affecting the toenails, are associated with poor tolerability or inadequate efficacy. Moreover, because many patients find onychomycosis embarrassing, achieving rapid cosmetic improvements is desirable. Kerasal Nail ${ }^{\mathrm{TM}}$ is a marketed topical treatment containing propylene glycol, urea and lactic acid. The ability of Kerasal Nail to improve the appearance of an affected nail was investigated in a 4-week open-label study. Patients aged $\geq 21$ years and with $25 \%$ to $75 \%$ of a big toenail or thumbnail (the "target" nail) affected by clinically suspected onychomycosis $(n=65)$ were instructed to apply Kerasal Nail to all their affected nails once daily for 4 weeks. After 1, 2 and 4 weeks of treatment, they assessed the appearance of the target nail on a 4-point scale, with a score of $\geq 2$ indicating a minimum of some improvement. They also assessed the nail for improvements in thickening, discoloration, brittleness and softness. Adverse events (AEs) occurring between administration of the first dose of Kerasal Nail and the end of the 4 -week treatment period were recorded. $85 \%$ of patients (95\% confidence interval [CI] 74 to 92 ) achieved some improvement in the target nail at 4 weeks. Improvement of the target nail was reported by $65 \%$ of patients (95\% CI 52 to 76 ) after 1 week of treatment and by $82 \%$ of patients (95\% CI 70 to 90 ) after 2 weeks. $55 \%$ of patients achieved some improvement in discoloration in the first week of treatment. After 4 weeks of treatment, discoloration, thickening, brittleness and softness were each improved in more than half of patients. Treatment-related AEs were limited to two cases of administration site pain. Overall treatment compliance was $99 \%$. In summary, Kerasal Nail produced very rapid improvements in nail appearance in patients with onychomycosis and was well tolerated.
\end{abstract}




\section{Keywords}

Onychomycosis, Kerasal Nail, Asian, Topical, 4 Weeks

\section{Introduction}

Onychomycosis, a fungal infection of the toenails and (less frequently) the fingernails, is characterized by discoloration of the nail plate, subungual hyperkeratosis/debris and onycholysis (detachment of all or part of the nail plate from the nail bed). It has been estimated to affect as many as a fifth of people in Europe and East Asia [1]. Its prevalence is higher in females than in males [2] and increases with age [1] [3].

Onychomycosis does not resolve spontaneously and if left untreated it can be transmitted to family members [4]. While not dangerous per se, it often causes pain and discomfort and can worsen health-related quality of life, particularly in patients with persistent symptoms [5]. It can be difficult to cure, with successful treatment taking up to 1 year [1]. Moreover, the relapse rate may be as high as $25 \%[6]$.

Treatment options for onychomycosis include laser therapy, removal of the affected nail by surgery or with chemicals, and various antifungal agents, applied topically or consumed systemically. Oral antifungals such as terbinafine and itraconazole are effective but cause unpleasant side effects such as headache, nausea, diarrhea and skin reactions [7]. They also have the potential for adverse liver effects and drug interactions, which may limit their use in the elderly-the population that is most likely to suffer from onychomycosis [8]. Conversely, topical agents such as amorolfine and ciclopirox, normally applied to the nail plate as lacquers, do not generally cause systemic toxicity but have inadequate efficacy because of their limited ability to penetrate the nail plate [9] [10].

Kerasal $\mathrm{Nail}^{\mathrm{TM}}$ is a marketed topical treatment containing propylene glycol, urea and lactic acid. In one early study, a different test solution containing these same three ingredients cured or yielded improvements in 21 of 23 patients with culture-positive onychomycosis when used for 2 to 6 months [11]. A subsequent 24-week randomized double-blind placebo-controlled study in 493 patients with distal subungual onychomycosis investigated K101, which is similar to Kerasal Nail but has a higher urea content (20\% vs 9.5\%) [12]. The mycological cure rate was higher for K101 versus placebo and a greater proportion of patients treated with $\mathrm{K} 101$ reported improvement in their disease.

Even after successful therapy with systemic or topical antifungals, affected nails may not have a normal appearance because clearance of signs of infection typically requires turnover of the nail plate [13]. Patients with onychomycosis often suffer from embarrassment and other psychosocial problems [14] [15] [16] and would therefore benefit from alternative treatments that rapidly enhance the cosmetic appearance of affected nails. Because approximately three-quarters of patients treated with $\mathrm{K} 101$ in the 24 -week study reported at least some im- 
provement by week 8 [12], a further study specifically investigated improvement in appearance after 8 weeks of K101 treatment in 75 patients with onychomycosis [17]. The proportion of patients who reported at least some improvement in an affected nail was $77 \%$ at 2 weeks, $88 \%$ at 4 weeks and $92 \%$ at 8 weeks. No adverse events (AEs) judged to be related to K101 were reported.

The aim of the present study was to investigate the effects of Kerasal Nail in terms of overall appearance and specific physical attributes during and after 4 weeks of treatment.

\section{Methods}

An open-label, single-arm baseline-controlled 4-week post-marketing clinical investigation to evaluate the ability of Kerasal Nail to provide rapid improvements in nail appearance in patients with onychomycosis was conducted at two sites in Singapore (a university hospital and a dermatology clinic) in 2016. Kerasal Nail is a clear colorless solution whose ingredients are propylene glycol, urea, lactic acid, disodium EDTA, glycerin, sodium hydroxide and water. It is supplied in a plastic tube with a silicone tip to facilitate application.

The final study protocol (dated 20 Oct 2015) and the patient information and consent form (version 1.1, dated 23 Dec 2015) were approved, in writing, by the National Healthcare Group (NHG) Domain Specific Review Board (DSRB) on 08 Jan 2016, before the first patient was enrolled in the study. The study was conducted in accordance with the Declaration of Helsinki and ICH E6 Good Clinical Practice.

Male and female subjects who fulfilled the following inclusion criteria were invited to participate: i) age $\geq 21$ years, ii) $25 \%$ to $75 \%$ of a big toenail or thumbnail (the "target" nail) affected by clinically suspected onychomycosis and (iii) willingness and ability to provide signed written informed consent. The following exclusion criteria were applied: i) proximal subungual onychomycosis, ii) other conditions known to cause an abnormal nail appearance, iii) topical treatment of the nail with an antifungal medication or systemic use of an antifungal treatment in the previous month, iv) participation in another clinical study of an investigational drug or device during the previous 4 weeks and v) known allergy to any of the ingredients in the study treatment.

The study comprised a screening visit and follow-up visits at 1, 2 and 4 weeks. At the screening visit, patients were assessed for demographics, medical history and prior and concomitant medications and underwent a physical examination of the hands and feet. They received instructions on how to use Kerasal Nail and applied it to all their affected nails under supervision. They were instructed to thereafter apply Kerasal Nail to all affected nails once daily at bedtime for 4 weeks. Compliance was assessed at each follow-up visit by asking patients how many days per week on average they had applied the study treatment since the previous study visit.

At screening, the target nail was selected for follow-up assessments. Images of the target nail were captured by standardized photography at the screening visit 
and the 1-, 2- and 4-week follow-up visits. After 1, 2 and 4 weeks of treatment, patients assessed the appearance of the target nail compared to baseline using a 4-point global assessment scale: 1 = No improvement, 2 = Some improvement, 3 = Clear improvement and $4=$ Very good improvement. They were also asked whether, compared to baseline, the target nail had become i) less thickened, ii) less discolored, iii) less brittle and iv) softer (Yes/No/Cannot determine).

The primary endpoint was the proportion of patients who experienced at least some improvement in the target nail after 4 weeks of treatment, based on a score of $\geq 2$ on the global assessment scale. The secondary endpoints were the proportions of patients who experienced i) at least some improvement in the target nail at 1 and 2 weeks and ii) improvements in thickening, discoloration, brittleness and softness at 1,2 and 4 weeks.

AEs that occurred between administration of the first dose of Kerasal Nail and the end of the 4-week treatment period, whether judged to be related to the study treatment or not, were recorded at each study visit. AEs were classified based on severity, seriousness and relationship to study treatment.

All patients who used the study product at least once were included in the safety set; those who additionally underwent at least one post-baseline assessment were included in the full analysis set (FAS). The per-protocol set (PPS) comprised patients who had i) at least $80 \%$ treatment compliance during the 4-week study period, ii) no major protocol deviations and iii) a score for the global assessment scale at 4 weeks. The primary efficacy analysis was based on the FAS; missing values were imputed by the last observation carried forward method. If a value for the 1-week follow-up visit was missing, the baseline value was imputed.

Based on previous studies of similar products, it was determined that a success rate of $50 \%$ with a $95 \%$ confidence interval of \pm 0.123 would be sufficient to confirm early visible improvements in target nail appearance (success being defined as a score of $\geq 2$ on the 4-point global assessment scale after 4 weeks of treatment). It was calculated that 65 patients would need to be enrolled to achieve this.

Statistical analyses were performed using STATA 14.0 (StataCorp LP, Texas, USA). Continuous data are presented as descriptive statistics: number of observations (n), mean and standard deviation (SD). Categorical data are presented as frequency (n) and percentage (\%). Two-sided 95\% confidence intervals (CIs) were computed using the Clopper-Pearson (exact) method. No adjustments were made for possible differences according to study site.

\section{Results}

68 subjects were screened and 65 of them were enrolled in the study. The first patient was enrolled on 19 Jan 2016 and the last patient completed the study on 15 Apr 2016. All 65 participants completed the study and were included in the FAS and safety set. Eight patients were excluded from the PPS because of inadequate treatment compliance $(n=1)$ or major protocol deviations $(n=7)$. Mean 
treatment compliance was $99 \%$ overall (median 100\%, range 76 to 100 ) and did not deteriorate over the course of the study (Table 1 ).

The mean age of participants was 53 years (median 53 years, range 26 to 86) and $29(45 \%)$ were female (Table 2). As shown in Table 3, 85\% of patients $(55 / 65)$ achieved at least some visible improvement in the target nail at 4 weeks (95\% CI 74 to 92). 65\% of patients (42/65) experienced some improvement of the target nail after 1 week of treatment (95\% CI 52 to 76 ) and $82 \%$ of them (53/65) experienced some improvement after 2 weeks (95\% CI 70 to 90). Figure 1 shows examples of improvements in a big toenail and a thumbnail during the 4-week treatment period.

More than half of patients achieved some improvement in discoloration in the first week of treatment (Table 4). Approximately half of patients showed reduced nail thickening by the end of the second week of treatment. After 4 weeks of treatment, discoloration, thickening, brittleness and softness were improved in more than half of patients (range $51 \%$ for brittleness to $72 \%$ for discoloration) (Table 4).

There were 3 AEs in 3 patients (5\%). All AEs were mild and none of them were serious. Two of the 3 AEs were pain at the administration site assessed as probably related to the study product; the other AE was a case of folliculitis judged not to be related to the study product. No patients discontinued treatment or were withdrawn from the study because of AEs.

Table 1. Treatment compliance according to information provided at the 1-, 2- and 4-week follow-up visits (safety set).

\begin{tabular}{cc}
\hline Treatment period & Compliance \\
Week $1^{\mathbf{a}}$ & $99.6(2.5)$ \\
Mean (SD) & 100 \\
Median & 86 to 100 \\
Range & \\
Week 2 & $98.7(9.0)$ \\
Mean (SD) & 100 \\
Median & 29 to 100 \\
Range & \\
Weeks 3 to 4 & $99.8(1.8)$ \\
Mean (SD) & 100 \\
Median & 86 to 100 \\
Range & 76 to 100 \\
Weeks 1 to 4 & $99.3(3.1)$ \\
Mean (SD) & 100 \\
Median &
\end{tabular}

${ }^{a}$ Data missing for one patient. 
Table 2. Demographics and baseline characteristics (full analysis set/safety set).

\begin{tabular}{cc}
\hline & $\mathrm{N}=65$ \\
\hline Age (years) & $52.7(13.0)$ \\
Mean (SD) & 53 \\
Median & 26 to 86 \\
Range & 0 \\
Missing (n) & \\
Sex, $\mathbf{n}(\%)$ & $29(45)$ \\
Female & $36(55)$ \\
Male & \\
Race, $\mathbf{n}(\%)$ & $64(98)$ \\
Asian or Pacific Islander & $1(2)$ \\
Caucasian & \\
Fingernails/toenails, $\mathbf{n}(\%)$ & $0(0)$ \\
Normal & $65(100)$ \\
Abnormal & \\
Skin on hands/feet, $\mathbf{n}(\%)$ & $57(88)$ \\
Normal & $8(12)$ \\
Abnormal & \\
\hline
\end{tabular}

Table 3. Changes in overall appearance of the target nail based on global assessment scale score (full analysis set, last observation carried forward).

\begin{tabular}{lccccccc}
\hline Appearance of target nail compared to baseline & \multicolumn{2}{c}{1 week } & \multicolumn{2}{c}{2 weeks } & \multicolumn{2}{c}{4 weeks } \\
\hline & $\mathrm{n}$ & $\%$ & $\mathrm{n}$ & $\%$ & $\mathrm{n}$ & $\%$ \\
\hline 1. No improvement & 23 & 35 & 12 & 18 & 10 & 15 \\
2. Some improvement & 34 & 52 & 36 & 55 & 29 & 45 \\
3. Clear improvement & 8 & 12 & 17 & 26 & 19 & 29 \\
4. Very good improvement & 0 & 0 & 0 & 0 & 7 & 11 \\
$\mathbf{2}$ At least some improvement & $\mathbf{4 2}$ & $\mathbf{6 5}$ & $\mathbf{5 3}$ & $\mathbf{8 2}$ & $\mathbf{5 5}$ & $\mathbf{8 5}$ \\
\hline
\end{tabular}

Percentage values are correct to the nearest whole number. The values for 1 week and 2 weeks add up to 99 (rather than 100) because of rounding.
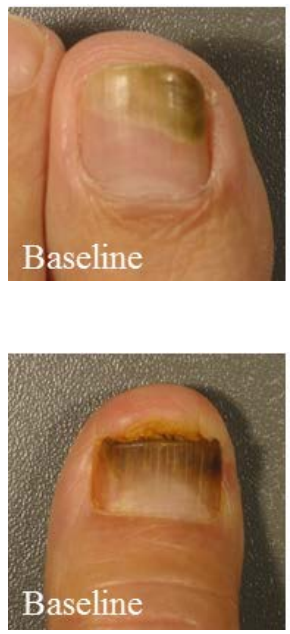
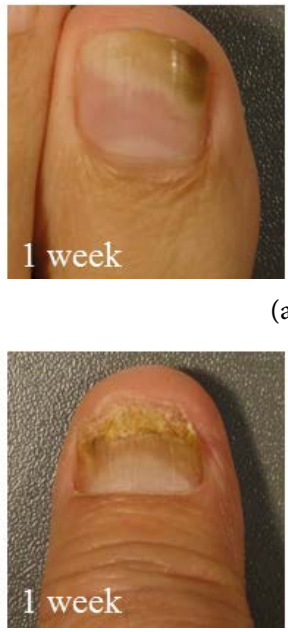

(a)
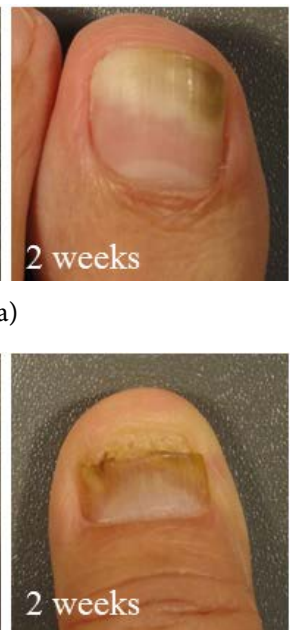

(b)
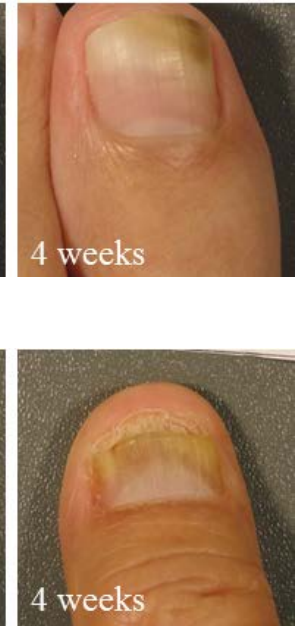

Figure 1. Visible improvements in target nails from baseline to 4 weeks. (a) A left big toenail. (b) A left thumbnail. 
Table 4. Proportions of patients experiencing improvements in individual target nail attributes after 1, 2 and 4 weeks of treatment (full analysis set, last observation carried forward).

\begin{tabular}{lccccccccc}
\hline \multicolumn{1}{c}{ Target nail attribute } & \multicolumn{3}{c}{1 week } & \multicolumn{2}{c}{2 weeks } & \multicolumn{2}{c}{4 weeks } \\
\hline & $\mathrm{n}$ & $\%$ & $95 \% \mathrm{CI}^{\mathrm{a}}$ & $\mathrm{n}$ & $\%$ & $95 \% \mathrm{CI}^{\mathrm{a}}$ & $\mathrm{n}$ & $\%$ & $95 \% \mathrm{CI}^{\mathrm{a}}$ \\
\hline Thickening & 18 & 28 & 17 to 40 & 34 & 52 & 40 to 65 & 45 & 69 & 57 to 80 \\
Discoloration & 36 & 55 & 43 to 68 & 45 & 69 & 57 to 80 & 47 & 72 & 60 to 83 \\
Brittleness & 19 & 29 & 19 to 42 & 30 & 46 & 34 to 59 & 33 & 51 & 38 to 63 \\
Softness & 23 & 35 & 24 to 48 & 31 & 48 & 35 to 60 & 41 & 63 & 50 to 75 \\
\hline
\end{tabular}

${ }^{\mathrm{a}}$ For the \% values.

\section{Discussion}

In this 4 -week open-label study, $85 \%$ of patients with onychomycosis reported at least some improvement in the appearance of an affected nail after treatment with Kerasal Nail for 4 weeks (the primary endpoint). 65\% of patients experienced visible improvement after just 1 week of treatment and $82 \%$ after 2 weeks. These values are comparable to those from the 8-week study of K101, in which $77 \%$ of patients reported at least some visible improvement at 2 weeks and $88 \%$ at 4 weeks [17]. As with K101 [17], improvements in thickening, discoloration, brittleness and softness were observed at 4 weeks, with discoloration the first physical attribute to show improvement in more than half of participants.

Previous studies have highlighted the embarrassment and stigmatization many onychomycosis patients suffer. In a US telephone survey of 258 patients with confirmed onychomycosis, $74 \%$ of participants reported a feeling of embarrassment [14]. Other studies describe low self-esteem and social isolation in patients [15]. In a recent online survey, 1017 Hong Kong residents were shown photographs of people with and without onychomycosis and asked questions about them. Respondents were less likely to engage in sporting or social activities with onychomycosis patients and more likely to feel uncomfortable in their presence [16]. Rapid cosmetic improvements such as those seen here with Kerasal Nail are important for onychomycosis patients to feel better about themselves and to avoid being or feeling stigmatized.

Because sustained treatment is typically needed to cure onychomycosis, adherence to therapy is very important. While the factors determining treatment adherence in onychomycosis are poorly understood, one study found that frequency of therapy was a key consideration for patients [18]. Another study reported that the main reasons for non-adherence include AEs and patients misinterpreting visible improvements as evidence that they were already cured [17]. In the present study, patient-rated improvements in affected nails were achieved with few treatment-related AEs. As with K101 [17], mean overall adherence was extremely high (99\%). This may both explain the rapid results and reflect the convenience and ease of use of Kerasal Nail.

The only treatment-related AEs in the present study were transient pain at the administration site in 2 patients. There were no treatment-related AEs in the 
8-week study of K101 [17], in which, as with Kerasal Nail in the present study, the treatment was applied using a silicone tip. The silicone tip may facilitate application to the nail, thereby limiting accidental contact with the adjacent skin. This in turn may prevent local irritation and pain such as that seen in $6 \%$ of participants in the 24-week study of K101 [12], in which K101 was applied dropwise.

Limitations of the present study include the lack of an active or placebo control group and the associated lack of blinding. Participants were aware that they were receiving an active treatment and this may have affected their scoring of the target nail. This is important given that all the study's efficacy variables were subjective. Also, the participants were recruited in Singapore at a single university hospital and a single dermatology clinic and may therefore not be representative of the spectrum of onychomycosis patients in South-East Asia or even Singapore. Furthermore, 64 of the 65 participants had "Asian or Pacific Islander" as their race, so the results of the present study may not necessarily be generalizable to patients of other races. However, the results were similar to those obtained in the 8-week study of K101 [17], which was conducted in Sweden. This indicates that Kerasal Nail is equally effective in populations of different ethnicities. Moreover, the patient sample in the present study covered both sexes and a wide age range ( 26 to 86 years).

\section{Conclusion}

In conclusion, Kerasal Nail elicited rapid effects in patients with onychomycosis, with two-thirds of patients reporting improvements in affected nails after just 1 week of treatment. The excellent overall treatment compliance suggests that the study participants felt motivated to adhere to treatment, possibly because of the effectiveness, tolerability and convenience of using Kerasal Nail. This is important because achieving optimal therapeutic results will typically take much longer compared to the early visible effects seen in this study. Users of Kerasal Nail should therefore be cautioned against discontinuing treatment prematurely.

\section{Acknowledgements}

P. J. Y. thanks Dr. Benson Yeo, Dr. Joel Lim and Dr. Teoh Yee Leng for helping to recruit patients, and Yan Qin, clinical trial coordinator at the National Skin Centre, Singapore. C. L. T. thanks Dr. Derrick Aw and Dr. Aditi Sobti for helping to recruit patients, and Mr. Nick Choi, clinical trial coordinator at the Division of Dermatology, National University Hospital, Singapore. A. T, on behalf of Moberg Pharma $A B$, thanks the patients and staff who participated in the study. The authors also acknowledge Stephen Gilliver of TFS AB for medical writing services, paid for by Moberg Pharma AB. Moberg Pharma AB funded the study and supplied the study product. Execution of the study, data analysis and reporting were outsourced to a contract resource organization.

\section{References}

[1] Ghannoum, M. and Isham, N. (2014) Fungal Nail Infections (Onychomycosis): A 
Never-Ending Story? PLoS Pathogens, 10, e1004105. https://doi.org/10.1371/journal.ppat.1004105

[2] Gupta, A.K., Jain, H.C., Lynde, C.W., Macdonald, P., Cooper, E.A. and Summerbell, R.C. (2000) Prevalence and Epidemiology of Onychomycosis in Patients Visiting Physicians' Offices: A Multicenter Canadian Survey of 15,000 Patients. Journal of the American Academy of Dermatology, 43, 244-248. https://doi.org/10.1067/mjd.2000.104794

[3] Elewski, B.E. and Charif, M.A. (1997) Prevalence of Onychomycosis in Patients Attending a Dermatology Clinic in Northeastern Ohio for Other Conditions. Archives of Dermatology, 133, 1172-1173. https://doi.org/10.1001/archderm.1997.03890450124022

[4] Tosti, A. and Elewski, B.E. (2016) Onychomycosis: Practical Approaches to Minimize Relapse and Recurrence. Skin Appendage Disorders, 2, 83-87. https://doi.org/10.1159/000448056

[5] Milobratović, D., Janković, S., Vukičević, J., Marinković, J., Janković, J. and Railić, Z. (2013) Quality of Life in Patients with Toenail Onychomycosis. Mycoses, 56, 543-551. https://doi.org/10.1111/myc.12072

[6] Scher, R.K. and Baran, R. (2003) Onychomycosis in Clinical Practice: Factors Contributing to Recurrence. British Journal of Dermatology, 149, 5-9. https://doi.org/10.1046/j.1365-2133.149.s65.5.x

[7] Gupta, A.K. and Shear, N.H. (2000) A Risk-Benefit Assessment of the Newer Oral Antifungal Agents Used to Treat Onychomycosis. Drug Safety, 22, 33-52. https://doi.org/10.2165/00002018-200022010-00004

[8] Elewski, B. and Tavakkol, A. (2005) Safety and Tolerability of Oral Antifungal Agents in the Treatment of Fungal Nail Disease: A Proven Reality. Therapeutics and Clinical Risk Management, 1, 299-306.

[9] Lauharanta, J. (1992) Comparative Efficacy and Safety of Amorolfine Nail Lacquer 2\% versus 5\% Once Weekly. Clinical and Experimental Dermatology, 17, 41-43. https://doi.org/10.1111/j.1365-2230.1992.tb00277.x

[10] Alley, M.R.K., Baker, S.J., Beutner, K.R. and Plattner, J. (2007) Recent Progress on the Topical Therapy of Onychomycosis. Expert Opinion on Investigational Drugs, 16, 157-167. https://doi.org/10.1517/13543784.16.2.157

[11] Faergemann, J. and Swanbeck, G. (1989) Treatment of Onychomycosis with a Propylene Glycol-Urea-Lactic Acid Solution. Mycoses, 32, 536-540. https://doi.org/10.1111/j.1439-0507.1989.tb02178.x

[12] Emtestam, L., Kaaman, T. and Rensfeldt, K. (2012) Treatment of Distal Subungual Onychomycosis with a Topical Preparation of Urea, Propylene Glycol and Lactic Acid: Results of a 24-Week, Double-Blind, Placebo-Controlled Study. Mycoses, 55, 532-540. https://doi.org/10.1111/j.1439-0507.2012.02215.x

[13] Scher, R.K., Tavakkol, A., Sigurgeirsson, B., Hay, R.J., Joseph, W.S., Tosti, A., Fleckman, P., Ghannoum, M., Armstrong, D.G., Markinson, B.C. and Elewski, B.E. (2007) Onychomycosis: Diagnosis and Definition of Cure. Journal of the American Academy of Dermatology, 56, 939-944. https://doi.org/10.1016/j.jaad.2006.12.019

[14] Drake, L.A., Scher, R.K., Smith, E.B., Faich, G.A., Smith, S.L., Hong, J.J. and Stiller, M.J. (1998) Effect of Onychomycosis on Quality of Life. Journal of the American Academy of Dermatology, 38, 702-704. https://doi.org/10.1016/S0190-9622(98)70199-9

[15] Chacon, A., Franca, K., Fernandez, A. and Nouri, K. (2013) Psychosocial Impact of Onychomycosis: A Review. International Journal of Dermatology, 52, 1300-1307. 
https://doi.org/10.1111/ijd.12122

[16] Chan, H.H., Wong, E.T. and Yeung, C.K. (2014) Psychosocial Perception of Adults with Onychomycosis: A Blinded, Controlled Comparison of 1017 Adult Hong Kong Residents with or without Onychomycosis. BioPsychoSocial Medicine, 8, 15.

https://doi.org/10.1186/1751-0759-8-15

[17] Faergemann, J., Gullstrand, S. and Rensfeldt, K. (2011) Early and Visible Improvements after Application of K101 in the Appearance of Nails Discoloured and Deformed by Onychomycosis. Journal of Cosmetics, Dermatological Sciences and Applications, 1, 59-63. https://doi.org/10.4236/jcdsa.2011.13010

[18] Nolting, S.K., Sanchez Carazo, S., De Boulle, K. and Lambert, J.R. (1998) Oral Treatment Schedules for Onychomycosis: A Study of Patient Preference. International Journal of Dermatology, 37, 454-456.

https://doi.org/10.1046/j.1365-4362.1998.00357.x

Submit or recommend next manuscript to SCIRP and we will provide best service for you:

Accepting pre-submission inquiries through Email, Facebook, LinkedIn, Twitter, etc. A wide selection of journals (inclusive of 9 subjects, more than 200 journals)

Providing 24-hour high-quality service

User-friendly online submission system

Fair and swift peer-review system

Efficient typesetting and proofreading procedure

Display of the result of downloads and visits, as well as the number of cited articles

Maximum dissemination of your research work

Submit your manuscript at: http://papersubmission.scirp.org/

Or contact jcdsa@scirp.org 